\title{
Contingent capture of involuntary visual attention interferes with detection of auditory stimuli
}

\author{
Marc R. Kamke* and Jill Harris \\ The Queensland Brain Institute, The University of Queensland, St. Lucia, QLD, Australia
}

\section{Edited by:}

Richard A. Abrams, Washington

University, USA

Reviewed by:

Mike Le Pelley, University of New

South Wales, Australia

Feng Du, Chinese Academy of

Sciences, China

${ }^{*}$ Correspondence:

Marc R. Kamke, The Queensland

Brain Institute, Building 79, The

University of Queensland, St. Lucia,

QLD 4072, Australia

e-mail:m.kamke@uq.edu.au
The involuntary capture of attention by salient visual stimuli can be influenced by the behavioral goals of an observer. For example, when searching for a target item, irrelevant items that possess the target-defining characteristic capture attention more strongly than items not possessing that feature. Such contingent capture involves a shift of spatial attention toward the item with the target-defining characteristic. It is not clear, however, if the associated decrements in performance for detecting the target item are entirely due to involuntary orienting of spatial attention. To investigate whether contingent capture also involves a non-spatial interference, adult observers were presented with streams of visual and auditory stimuli and were tasked with simultaneously monitoring for targets in each modality. Visual and auditory targets could be preceded by a lateralized visual distractor that either did, or did not, possess the target-defining feature (a specific color). In agreement with the contingent capture hypothesis, target-colored distractors interfered with visual detection performance (response time and accuracy) more than distractors that did not possess the target color. Importantly, the same pattern of results was obtained for the auditory task: visual target-colored distractors interfered with sound detection. The decrement in auditory performance following a target-colored distractor suggests that contingent capture involves a source of processing interference in addition to that caused by a spatial shift of attention. Specifically, we argue that distractors possessing the targetdefining characteristic enter a capacity-limited, serial stage of neural processing, which delays detection of subsequently presented stimuli regardless of the sensory modality.

Keywords: selective attention, contingent capture, spatial attention, bottleneck, visual, auditory

\section{INTRODUCTION}

It is commonly held that the human brain is equipped with selection mechanisms that filter and prioritize incoming sensory signals. Known as selective attention, these mechanisms can be captured involuntarily by highly salient events in the environment, or directed voluntarily according to goals or task demands (for review, see Yantis, 2000). The involuntary capture of visual attention, however, can also be influenced by the goals of an observer. Specifically, when attention is set to select an item with a particular characteristic, such as a specific color, irrelevant items possessing that property will involuntarily capture attention to a greater degree than items not sharing the feature (such as different colored or moving items; see Folk et al., 1992, 1994). For example, waiting at an intersection for a green signal, a driver may be seen to lurch forward following the appearance of a green turning arrow. Despite appearing in the wrong spatial location and being the wrong shape, the arrow captures the driver's attention due to its (task-relevant) color (Ghorashi et al., 2003). Converging evidence suggests that such contingent capture involves a shift of spatial attention toward the item containing the target-defining feature, but it is not clear if the associated decrements in performance for detecting a target item are entirely due to involuntary orienting of spatial attention. Here, we address this question by determining whether the detection of auditory events is hindered during contingent capture of visual spatial attention.
There is now a wealth of evidence suggesting that involuntary contingent capture involves a shift in visuospatial attention. For example, behavioral studies have shown that irrelevant distractors that share the target's defining characteristic spatially cue targets (Folk et al., 1992, 1994; Bacon and Egeth, 1994; Gibson and Kelsey, 1998) and improve identification when accompanied by a perceptual prime for the target (Folk et al., 2002). Importantly, equally salient distractors that do not share the target's defining characteristic do not cause such cueing or priming effects. Using functional magnetic resonance imaging (fMRI) it has been shown that, compared to non-target colored distractors, target-colored distractors are associated with increased neural activity in parietal brain regions putatively involved in spatial attention (Serences et al., 2005). Similarly, electroencephalography studies have shown that contingent capture is associated with an enhanced N2pc event-related potential, which is believed to index a shift in visuospatial attention (e.g., Eimer and Kiss, 2008; Kiss etal., 2008; Leblanc etal., 2008; Lien et al., 2008; Ansorge etal., 2009, 2011; Brisson etal., 2009). In one compelling demonstration, targets were always presented within a central stream of stimuli whilst distractors were presented to the side of the central stream (Leblanc etal., 2008). Despite the fact that distractors were irrelevant to the task and never appeared at the target location, target-colored, but not nontarget-colored distractors were associated with a decrement in 
behavioral performance and induced a shift in visuospatial attention, as indexed by the N2pc (for a similar result, see Jolicoeur et al., 2006).

Although there is strong evidence showing that the involuntary capture of visual spatial attention is contingent upon the behavioral goals of the observer (but see, e.g., Theeuwes, 2010), it is not clear if the associated decrements in behavioral performance are entirely due to orienting of spatial attention. Evidence for an additional interference is gained from studies showing that irrelevant distractors possessing the target-defining feature interfere with target detection even when shifts in visuospatial attention were prevented by presenting distractors at fixation (e.g., Ghorashi et al., 2003; Folk et al., 2008). To explain these effects Ghorashi and colleagues proposed a two-stage model in which stimuli must first pass an input filter that is tuned to the target's defining feature before gaining access to a capacitylimited stage of processing that is serial in nature. Importantly, access to this second stage is unavailable while a distractor is being processed, thereby interfering with processing of the target itself.

If capacity-limited central resources add to the behavioral decrements observed with contingent capture, then processing of an irrelevant distractor item should interfere not only with detection of the associated target, but also with other tasks requiring central resources. Preliminary support for this hypothesis can be found in a study in which a concurrent auditory discrimination task was used to investigate the influence of cognitive load on contingent capture (Brisson et al., 2009). In that study, N2pc data revealed that the capture of visuospatial attention by targetcolored distractors was reduced when participants undertook a demanding auditory task (Brisson et al., 2009). Interestingly, performance on the auditory task also varied across the distractor conditions, being poorest for target-colored items. The difference was, however, very small (albeit statistically significant; 83-86\% across all conditions) and complicated by a speed-accuracy trade off. Prompted by this incidental observation, instead of asking if a secondary task influences contingent capture we investigated whether contingent capture influences cognitive processing of a secondary task. We hypothesized that if visual items that capture spatial attention enter a capacity-limited, serial stage of neural processing, then behavioral performance will be negatively impacted for both visual and auditory targets that appear while the distractor is being processed. We tested this hypothesis by investigating the influence of irrelevant visual distractors on visual and auditory target detecting. Distractors included an item that shared its color with the to-be-detected visual target as well as a non-target-colored item. To provide an additional test of the contingent capture effects a third distractor, which rotated but did not change color, was included.

\section{MATERIALS AND METHODS PARTICIPANTS}

Twenty-four adult volunteers participated in the study (median age 32 years; range 23-53 years; 16 female). All participants had normal or corrected-to-normal vision and no known hearing problems. Study procedures were approved by the Medical Research Ethics Committee at The University of Queensland and fully informed, written consent was obtained from all participants.

\section{STIMULI}

Visual stimuli consisted of six digits of similar luminance $(2,3$, 5, 6, 8, and 9; all within $1 \mathrm{~cd} / \mathrm{m}^{2}$; ColorCAL colorimeter, Cambridge Research Systems) that were colored blue (RGB: 0, 0, 225), green $(0,115,0)$, purple $(140,0,170)$, red $(213,0,0)$, or ochre $(149,79,0)$. As shown in Figure 1, the digits were presented in a rapid stream at the center of a computer screen and were flanked $2^{\circ}$ to the left and right by a gray $(85,85,85)$ hash symbol. All stimuli were presented in Arial font on a black background and subtended $1.3^{\circ}$ in height. Each stimulus was presented for $117 \mathrm{~ms}$ with a stimulus onset asynchrony of $150 \mathrm{~ms}$. Visual targets were the number " 3 " or " 8 " presented in either blue, green, purple or red. Each participant was assigned a single target color for the duration of the experiment, but colors were counterbalanced across participants. A visual target was presented on $50 \%$ of trials and occurred randomly at position 9,12 , or 15 in the stream. Targets were always followed by nine non-target digits. Non-target digits were randomly generated from all numbers used (including " 3 " and " 8 ") and all non-target colors.

Auditory stimuli were presented free field at $\sim 70 \mathrm{~dB}$ SPL (measured at the ear; Bruel and Kjaer, 2205) using desktop speakers placed directly to either side of the display screen. The "standard" auditory stimulus was a $1000 \mathrm{~Hz}$ pure tone with $70 \mathrm{~ms}$ duration, which was presented simultaneously with every third digit in the visual stream (see Figure 1). Auditory targets consisted of one of two "oddball" tones, one lower and one higher in frequency than the standard tone. To provide a perceptually equivalent difference between higher and lower oddballs the target tones differed from the standard by the same frequency ratio. Furthermore, to ensure that the difficulty of the auditory discrimination task was similar across participants, different high and low oddball tones were used depending on accuracy in a titration task (see Procedure). Targets had the following frequency ratio relative to the $1000 \mathrm{~Hz}$ standard tone: 1.4 (a high tone of $1400 \mathrm{~Hz}$ and a low tone $714 \mathrm{~Hz}), 1.2(1200$ and $833 \mathrm{~Hz}), 1.1(1100$ and $909 \mathrm{~Hz}), 1.05(1050$ and $952 \mathrm{~Hz}), 1.025(1025$ and $976 \mathrm{~Hz})$, and $1.01(1010$ and $990 \mathrm{~Hz})$. Thus, the ratio 1.01 was the most difficult to discriminate and the ratio 1.4 was the easiest. An auditory target was presented on the $50 \%$ of trials in which there was no visual target. Auditory targets were accompanied by both lateral hash marks, but no central digit. The central digit was not concurrently presented with an auditory target so that the task could be adapted for an evoked potential study in future research. Importantly, the central digit was absent whenever an auditory target was presented (i.e., following the no-distractor and all distractor conditions), so any differences in behavioral performance across the visual distractor conditions cannot be attributed to this factor.

On each trial, immediately prior to the appearance of a visual or auditory target one of either three visual distractors, or no distractor, was presented (in equal proportions). As shown in Figure 1 (insert), distractors consisted of either one of the hash marks changing to the target color (target-colored distractor), one of the hash marks changing to a non-target color (non-target-colored 


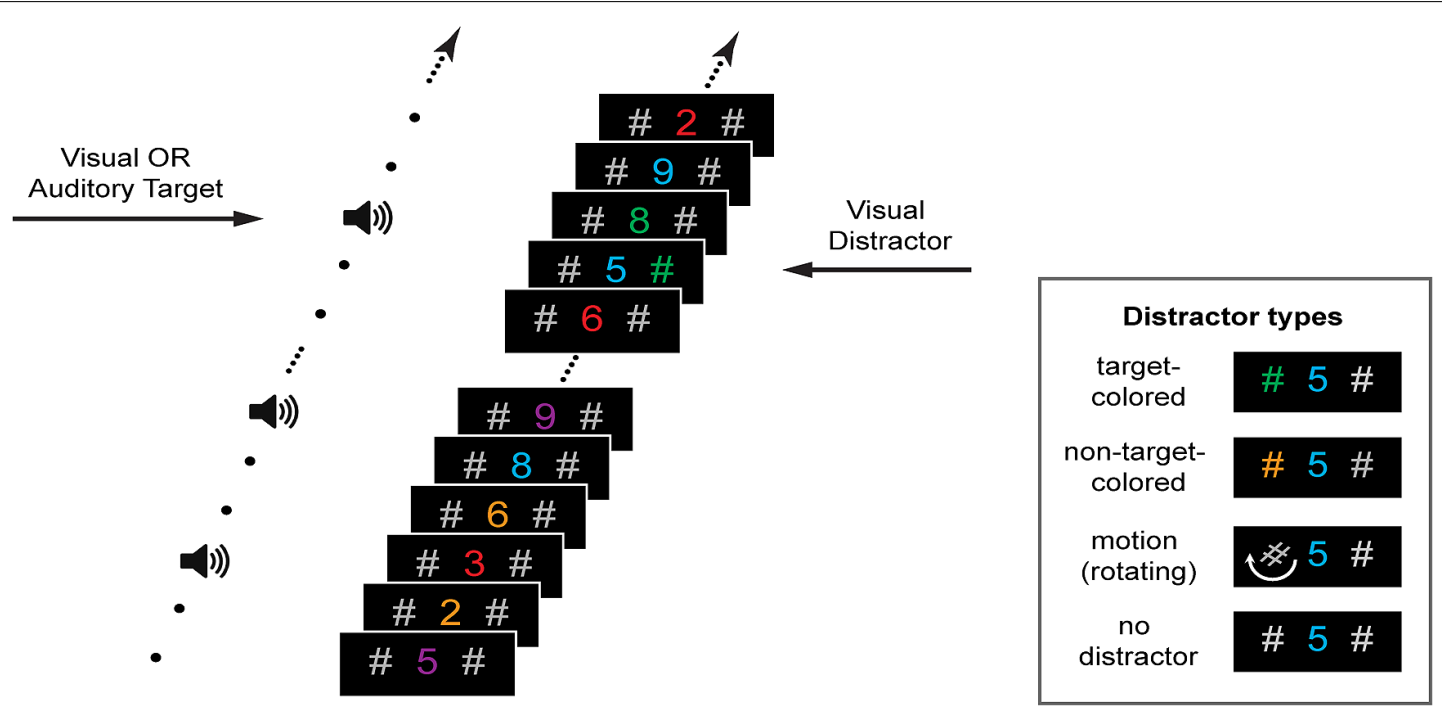

FIGURE 1 | Stimuli and trial design. Participants fixated a central stream of rapidly presented digits and listened to tones. Their task was to detect either a number presented in a pre-defined color or a change in the tonal frequency. Prior to the presentation of a visual or auditory target $(50 \%$ probability) either no distractor or one of three visual distractors was presented on the left or right of fixation. Distractors matched the color of the visual target, were in a different color, or did not change color but rotated (see insert). distractor), or one of the hash marks rotating $540^{\circ}$ over the display period (motion distractor). Distractors were equally likely to appear on the left and right sides of the central stream. On 25\% of trials only the gray hash marks were presented ("no-distractor" control condition). Stimulus presentation was controlled using Presentation software (v14, Neurobehavioral Systems) running under Windows Vista on a laptop computer with a 17" LCD display (70 cm viewing distance) and a refresh rate of $60 \mathrm{~Hz}$.

\section{PROCEDURE}

Prior to the start of the experiment participants were given practice trials of the visual task alone (no auditory stimuli presented), the auditory task alone and then the full task. To ensure that performance on the auditory task was comparable between participants, and to avoid floor and ceiling effects, an auditory titration task preceded the practice trials. The titration task consisted of eight high and eight low oddball tones from each of the six ratio levels (see Stimuli), presented randomly in two blocks. The oddball pair used in the experiment was the most difficult to discriminate in which the participants' accuracy was at least $80 \%$. Nine participants completed the most difficult discrimination (1.01 ratio), 10 the 1.025 ratio, 1 the 1.1 ratio, and 2 participants each completed the 2 easiest oddball pairs (1.2 and 1.4 ratio).

The experiment proper consisted of six blocks of 48 trials; 36 trials for each of the two target (visual or auditory) and four distractor conditions. Experimental trials commenced with a fixation cross followed by the concurrent presentation of the visual and auditory streams (see Figure 1). The participant was tasked with detecting, as quickly and accurately as possible, the number presented in a pre-defined target color or a change in tonal frequency. A four-alternative forced choice response was made via key press on a standard keyboard, which was labeled " 3 " or " 8 " for visual targets and "LOW" or "HI" for auditory targets. For half of the participants in each group, the visual response buttons were the D (" 3 ") and F (" 8 ") keys, pressed using the left hand, while the auditory response buttons were the J ("LOW") and $\mathrm{K}$ ("HI") keys, pressed using the right hand. The remaining participants in each group used the $\mathrm{J}$ (" 3 ") and $\mathrm{K}$ (" 8 ") keys for response to visual stimuli, and the D ("LOW") and F ("HI") keys for auditory responses. The four targets were presented with equal probability. Feedback on accuracy was presented after each trial. Within each condition, trial length and distracter position (right or left) were counterbalanced. Self-paced rest breaks were offered between blocks.

\section{STATISTICAL ANALYSIS}

Reaction times greater than 2.5 standard deviations from the mean for each condition, or that were less than $100 \mathrm{~ms}$, were removed prior to analysis. This screening procedure eliminated less than $2.2 \%$ of the data (range across participants: 0.4$3.7 \%$ ). Responses within each modality were then collapsed across the two target types (i.e., " 3 " or " 8 " for visual and "Low" or "High" for auditory). Differences in baseline behavioral performance between the visual and auditory tasks were investigated by comparing reaction times and accuracy in the no-distractor condition using two-tailed t-tests. To investigate contingent capture effects, individual responses for each distractor condition were expressed relative to the no-distractor baseline condition (i.e., visual distractor condition minus no-distractor condition). These difference data were then compared using repeated measures ANOVA with the factors distractor (target-colored, non-targetcolored, motion) and Task (visual, auditory). Significant main effects were followed-up using planned comparisons between the three distractor conditions using Bonferroni adjusted, twotailed $t$-tests (corrected alpha level $=0.017$ ). Because reaction 
time data were generally moderately positively skewed and accuracy data negatively skewed, but to a smaller extent, data were normalized using a $\log 10$ and square root (with reflection) transformation, respectively. The results of inferential analysis are based on this normalized data, but descriptive statistics are reported using the untransformed values. These transformations did not change the significance of any of inferential analyses. Statistical analyses were carried out using SPSS (v19; IBM).

\section{RESULTS}

Mean reaction times in the control (no distractor) condition were faster for visual $(632 \pm 17 \mathrm{~ms} ; M \pm \mathrm{SE})$ compared to auditory $(854 \pm 59 \mathrm{~ms})$ targets (mean difference $=-0.1157,95 \%$ $\mathrm{CI}=-0.1647$ to $\left.-0.0667 ; t_{23}=-4.886, p<0.001\right)$. Accuracy in the no-distractor condition was high and did not vary between the visual $(96.3 \pm 0.9 \%)$ and auditory $(93.3 \pm 1.9 \%)$ tasks (mean difference $=-0.3759,95 \% \mathrm{CI}=-1.1092$ to $0.3575 ; t_{23}=-1.060$, $p>0.29)$.

Figure 2 illustrates the influence of visual distractors on reaction times for both the visual and auditory tasks, relative to the no-distractor condition. For the visual task non-targetcolored and motion distractors had little influence on reaction times, whereas the same distractors were associated with a substantial speeding of auditory responses (negative change in response time). Critically, for both tasks the slowest responses occurred in the target-colored distractor condition. Repeated measures ANOVA confirmed that reaction times varied across the distractor conditions (Distractor: $F_{2,46}=7.353, p=0.002$; $\left.\eta_{\mathrm{p}}^{2}=0.242\right)$, but not between the visual and auditory tasks (Task: $F_{1,23}=2.330, p>0.14$; Distractor $\times$ Task: $F_{2,46}=1.135$, $p>0.32$ ). Specifically, reaction times were longer following the presentation of a target-colored distractor compared to both the non-target-colored $\left(t_{23}=2.875, p=0.009\right)$ and motion $\left(t_{23}=3.113, p=0.005\right)$ distractors. There was no difference between the non-target-color and motion conditions $\left(t_{23}=0.546\right.$, $p>0.58)$.

Figure 3 illustrates changes in accuracy for detecting visual and auditory targets, relative to the no-distractor condition. In general, accuracy was reduced in the presence of a visual distractor, with the highest reduction in the target-colored distractor condition. A repeated measures ANOVA confirmed that the change in accuracy varied across the distractor conditions (Distractor: $\left.F_{2,46}=4.568, p=0.016 ; \eta_{p}^{2}=0.166\right)$, but that there was no difference between the visual and auditory tasks (Task: $F_{1,23}=1.086$, $p>0.30$; Distractor $\times$ Task: $F_{2,46}=0.327, p>0.72$ ). Follow-up comparisons revealed that accuracy was lower in the target-colored compared to non-target-colored $\left(t_{23}=2.740, p=0.012\right)$, but not motion $\left(t_{23}=1.964, p=0.062\right)$, condition. There was no difference between the non-target-color and motion conditions $\left(t_{23}=-1.038, p>0.30\right)$.

\section{DISCUSSION}

There is now a wealth of evidence showing that the extent to which visual events involuntarily capture attention depends on the goals (attentional set) of an observer. In the present study participants simultaneously monitored streams of visual and auditory

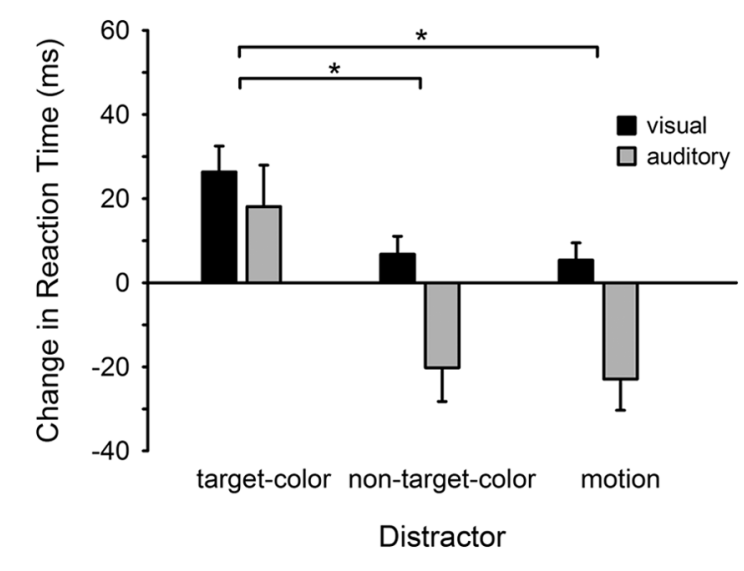

FIGURE 2 | Change in reaction time for detecting visual and auditory targets. Reaction times are plotted for each distractor condition relative to the baseline (no-distractor) condition. Reaction times were delayed following the presentation of a target-colored distractor for both the visual and auditory tasks. ${ }^{*} p<0.05$ (corrected for multiple comparisons). Error bars show within subjects (normalized) SEM (Cousineau, 2005).

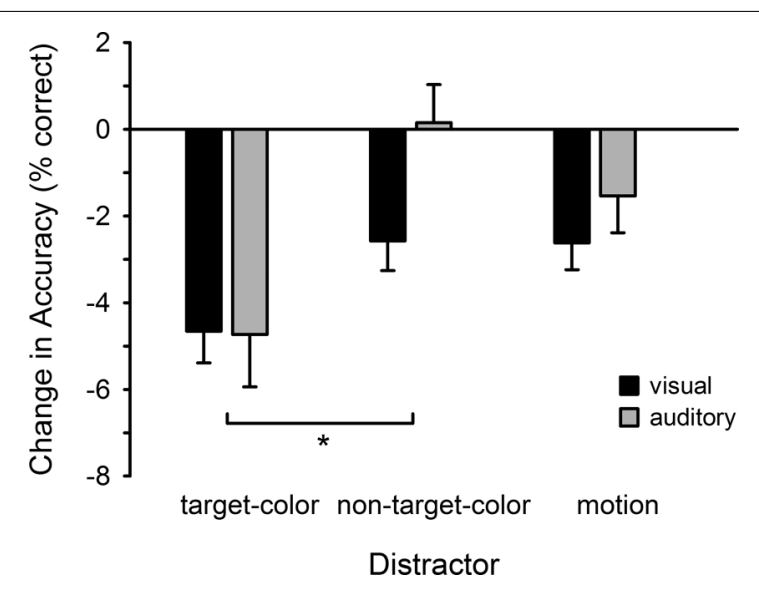

FIGURE 3 | Change in accuracy for detecting visual and auditory targets. Accuracy is plotted for each distractor condition relative to the baseline (no-distractor) condition. Changes in accuracy were identical for the visual and auditory tasks. ${ }^{*} p<0.05$ (corrected for multiple comparisons). Error bars show within subjects SEM.

stimuli for target items (digits of a particular color and atypical tones, respectively) while irrelevant visual distractors were presented to the left or right of fixation. Behavioral performance in the visual detection task was negatively impacted by distractors that possessed the target-defining characteristic compared to other distractors. Thus, the present results are consistent with the contingent capture hypothesis, which posits that involuntary capture of attention is influenced by attentional set (Folk et al., 1992, 1994). The novel observation is that the presentation of a visual distractor that possessed a target-defining feature also induced deficits in auditory target detection. Specifically, auditory performance was poorer following the presentation of a target-colored visual distractor compared to presentation of non-target colored and motion distractors. These results show that visual contingent 
capture affects processing not only of visual, but also auditory stimuli.

In the present study response times for detecting visual and auditory targets were significantly increased in the presence of a target-colored distractor compared to both non-target colored and motion distractors. The primary measure of interest was response time, as accuracy in the four-alternative forced-choice detection task was expected to be high. Nonetheless, for both the visual and auditory tasks the target-colored distractor was also associated with a reduction in accuracy compared to the non-target-colored condition, while there was no change in accuracy compared to the motion condition. This pattern of results suggests that the increase in reaction time following the presentation of a targetcolored distractor, relative to the other distractors, was not merely due to a speed-accuracy trade off. Importantly, the results also cannot be attributed to differences across the distractor conditions in salience at the sensory level, as target and non-target colors were of equal luminance and, across participants, were the same colors.

In a previous study using a paradigm similar to that employed here it was shown that the target-colored distractor induced a shift of visuospatial attention to the distractor location, as indexed by the N2pc event-related potential (Leblanc et al., 2008). The present results suggest that, as well as capturing visuospatial attention, irrelevant visual stimuli that possess the characteristic feature of a to-be-detected visual target can interfere with auditory processing. It is well established that visual stimuli appearing at a certain location can enhance detection of auditory stimuli appearing at that location and hamper detection of sounds presented elsewhere (for review, see Spence and McDonald, 2004). Such effects of cross-modal spatial attention, however, are unlikely to account for the current results as the auditory stimulus was not spatially distinct. Indeed, the visual distractor was located only $2^{\circ}$ from fixation, so any accompanying shift in visuospatial attention occurred within the bilateral auditory sources and below the spatial resolution of cross-modal cueing effects (e.g., Focker et al., 2010). Thus, our observed decrement in auditory performance following a target-colored distractor suggests that contingent capture involves a source of processing interference in addition to that caused by a spatial shift of attention.

It has been suggested that as well as causing a shift in visuospatial attention, contingent capture may induce a non-spatial capture of attention (Ghorashi et al., 2003). Initial support for this idea was garnered from the attentional blink phenomenon, which describes the decrement in detection of a second target (T2) when it appears soon after a first (T1; Ghorashi et al., 2003; Folk et al., 2008). In particular, it was shown that even when there was no requirement to report $\mathrm{T} 1$, detection of $\mathrm{T} 2$ was impaired if T1 shared critical features with the second target (Chun, 1997). Although the exact mechanisms have yet to be elucidated, it is commonly held that the attentional blink reveals a central bottleneck in neural processing (e.g., Raymond et al., 1992). Specifically, when T1 is detected it enters a capacity-limited, serial stage of processing that prevents or delays processing of T2 (for recent reviews, see Dux and Marois, 2009; Martens and Wyble, 2010). Further evidence supporting the idea that contingent capture may also involve a central bottleneck is provided by the observation that the N2pc component elicited by target-colored distractors was reduced when participants undertook a concurrent auditory task, which taxed central resources (Brisson et al., 2009). Moreover, it was recently reported that contingent capture is reduced or eliminated during the attentional blink (Du et al., 2013), suggesting that both phenomena depend on capacity-limited central resources. The notion that contingent capture involves such central resources can also account for the impairment we observed in auditory detection following the target-colored distractor.

Although we did not find any difference in the pattern of contingent capture effects between the visual and auditory detection tasks, data presented in Figure 2 show that the presence of nontarget-colored and motion distractors was associated with faster responses to auditory targets relative to the no-distractor condition. One explanation for this result is that the visual distractors may have acted as an alerting cue, as they were temporally predictive of a target. Critically, even if distractors had an alerting value this effect was identical across the distractor conditions and therefore cannot explain the selective slowing of responses to auditory targets associated with target-colored distractors. Moreover, it has been shown that eliminating the predictiveness of a distractor display that was similar to the one used here did not alter visual contingent-capture or the N2pc (Experiment 3, Leblanc et al., 2008). Nonetheless, if distractors did carry an alerting value then it could be argued that any cueing effect should manifest for all distractors in both the visual and auditory detection tasks. In this context it is possible that non-target-colored and motion distractors did facilitate responses to the auditory and visual targets, but for the latter any facilitation was counteracted by an involuntary shift of visuospatial attention toward the distractor item (Hickey et al., 2006). On this argument target-colored distractors presumably also cued (speeded) detection, however, that item would have gained access to a capacity-limited, serial stage of processing that interfered with identification of both visual and auditory targets.

Another interesting observation in the present study is that the absolute cost in response time following the presentation of a target-colored distractor, relative to the non-target-colored distractors, was numerically twice as large in the auditory task as it was in the visual task (see Figure 2). A similar result was reported previously when participants were required to maintain two attentional sets for color (such as for green and orange). In that study identification of a (e.g., orange colored) target was poorest following presentation of a distractor that matched the other attentional set (green; Moore and Weissman, 2010). The authors proposed that contingent capture involves not only a reduction in accuracy due to capacity-limited resources processing the distractor, but also an enhancement due to the attentional set entering the focus of attention (Moore and Weissman, 2011). Thus, when two attention sets must be maintained a target-colored distractor can interfere with detection not only by occupying central processing resources but also by impairing the ability to attend to a subsequent item whose color matches a different attentional set (Moore and Weissman, 2010). In line with this argument it is possible that the target-colored distractor in the present study captured 
visuospatial attention, allowing that item to enter a capacitylimited stage of processing that interfered with detection of the visual target. In addition, the target-colored distractor also lead to the corresponding attentional set for color entering the focus of attention, further restricting the capacity to process the auditory stimulus as its features were associated with a different attentional set.

Although our results are consistent with the notion that contingent capture involves an amodal bottleneck in neural processing, it could be argued that the target-colored distractor may have primed the hand that was used to respond to visual stimuli. For auditory targets such priming could have led to longer reaction times because the other hand was required to make a response. Importantly, because the distractor was not predictive of the target's modality or identity, such an effect could not have arisen due to participants intentionally using the distractor as a cue. Indeed, the distractor-to-target delay was likely too short to allow for intentional response preparation (Adam et al., 2005). Thus, any response priming was automatic and was also contingent upon attentional set, as it was specific to the target-colored distractor. In this context it should be noted that although participants searched for a colored item, their task was to report the identity of that item. Discrimination judgments of this sort, which are orthogonal to the dimension along which the cue varies, typically avoid response priming (for a review of cross-modal effects, see Spence et al., 2004). Thus, it seems unlikely that the target-colored distractor simply primed the relevant hand. It remains possible, however, that the target-colored distractor cued the stimulus-response mapping for visual targets (e.g., Mattler, 2006; Reuss et al., 2011), which could induce a cost when a switch to the auditory attention set (and its stimulus-response mapping) was required (Du et al., 2014). This argument is similar to our contention that the target-colored distractor brought into focus an attentional set for color, but emphasizes more the response-selection rather than discrimination stage of processing. Future research will be needed to determine the extent to which visual contingent capture affects the perception, decision, and response stages of sound detection.

In summary, we have shown that contingent capture by an irrelevant visual stimulus that matches top-down attentional control settings interferes with detection of both visual and auditory targets. The current results are consistent with the hypothesis that, in addition to a shift in spatial attention, contingent capture involves a serial stage of neural processing that is limited in capacity. More generally, the results are consistent with the notion that attentional selection acts to prioritize and restrict access to this capacity-limited processing stage.

\section{AUTHOR CONTRIBUTIONS}

Conceived and designed the experiments: Marc R. Kamke, Jill Harris. Acquired the data: Jill Harris. Analyzed the data: Marc R. Kamke, Jill Harris. Wrote and/or revised the paper: Marc R. Kamke, Jill Harris.

\section{ACKNOWLEDGMENTS}

The authors wish to thank Oscar Jacoby for programming assistance and feedback on the paper. This work was supported by a project grant from The Garnett Passe and Rodney Williams Memorial Foundation. The funders had no role in study design, data collection and analysis, decision to publish or preparation of the manuscript. The content is solely the responsibility of the authors and does not necessarily represent the official views of the Foundation.

\section{REFERENCES}

Adam, J., Hommel, B., and Umiltà, C. (2005). Preparing for perception and action (II): automatic and effortful processes in response cueing. Vis. Cogn. 12, 14441473. doi: 10.1080/13506280444000779

Ansorge, U., Kiss, M., and Eimer, M. (2009). Goal-driven attentional capture by invisible colors: evidence from event-related potentials. Psychon. Bull. Rev. 16, 648-653. doi: 10.3758/PBR.16.4.648

Ansorge, U., Kiss, M., Worschech, F., and Eimer, M. (2011). The initial stage of visual selection is controlled by top-down task set: new ERP evidence. Atten. Percept. Psychophys. 73, 113-122. doi: 10.3758/s13414-010-0008-3

Bacon, W. F., and Egeth, H. E. (1994). Overriding stimulus-driven attentional capture. Percept. Psychophys. 55, 485-496. doi: 10.3758/BF032 05306

Brisson, B., Leblanc, E., and Jolicoeur, P. (2009). Contingent capture of visualspatial attention depends on capacity-limited central mechanisms: evidence from human electrophysiology and the psychological refractory period. Biol. Psychol. 80, 218-225. doi: 10.1016/j.biopsycho.2008.10.001

Chun, M. M. (1997). Temporal binding errors are redistributed by the attentional blink. Percept. Psychophys. 59, 1191-1199. doi: 10.3758/BF03214207

Cousineau, D. (2005). Confidence intervals in within-subject designs: a simpler solution to Loftus and Masson's method. Tutor. Quant. Methods Psychol. 1, 42-45.

Du, F., Yang, J., Yin, Y., Zhang, K., and Abrams, R. A. (2013). On the automaticity of contingent capture: disruption caused by the attentional blink. Psychon. Bull. Rev. 20, 944-950. doi: 10.3758/s13423-013-0410-7

Du, F., Zhang, K., and Abrams, R. A. (2014). Hold the future, let the past go: Attention prefers the features of future targets. Cognition 131, 205-215. doi: 10.1016/j.cognition.2013.12.015

Dux, P. E., and Marois, R. (2009). The attentional blink: a review of data and theory. Atten. Percept. Psychophys. 71, 1683-1700. doi: 10.3758/APP.71.8.1683

Eimer, M., and Kiss, M. (2008). Involuntary attentional capture is determined by task set: evidence from event-related brain potentials. J. Cogn. Neurosci. 20, 1423-1433. doi: 10.1162/jocn.2008.20099

Focker, J., Hotting, K., Gondan, M., and Roder, B. (2010). Unimodal and crossmodal gradients of spatial attention: evidence from event-related potentials. Brain Topogr. 23, 1-13. doi: 10.1007/s10548-009-0111-8

Folk, C. L., Leber, A. B., and Egeth, H. E. (2002). Made you blink! Contingent attentional capture produces a spatial blink. Percept. Psychophys. 64, 741-753. doi: 10.3758/BF03194741

Folk, C. L., Leber, A. B., and Egeth, H. E. (2008). Top-down control settings and the attentional blink: evidence for nonspatial contingent capture. Vis. Cogn. 16, 616-642. doi: 10.1080/13506280601134018

Folk, C. L., Remington, R. W., and Johnston, J. C. (1992). Involuntary covert orienting is contingent on attentional control settings. J. Exp. Psychol. Hum. Percept. Perform. 18, 1030-1044. doi: 10.1037/0096-1523.18.4.1030

Folk, C. L., Remington, R. W., and Wright, J. H. (1994). The structure of attentional control: contingent attentional capture by apparent motion, abrupt onset, and color. J. Exp. Psychol. Hum. Percept. Perform. 20, 317-329. doi: 10.1037/00961523.20.2.317

Ghorashi, S. M., Zuvic, S. M., Visser, T. A., and Di, L. V. (2003). Focal distraction: spatial shifts of attentional focus are not required for contingent capture. J. Exp. Psychol. Hum. Percept. Perform. 29, 78-91. doi: 10.1037/0096-1523.29.1.78

Gibson, B. S., and Kelsey, E. M. (1998). Stimulus-driven attentional capture is contingent on attentional set for displaywide visual features. J. Exp. Psychol. Hum. Percept. Perform. 24, 699-706. doi: 10.1037/0096-1523.24.3.699

Hickey, C., McDonald, J. J., and Theeuwes, J. (2006). Electrophysiological evidence of the capture of visual attention. J. Cogn. Neurosci. 18, 604-613. doi: 10.1162/jocn.2006.18.4.604

Jolicoeur, P., Sessa, P., Dell'Acqua, R., and Robitaille, N. (2006). Attentional control and capture in the attentional blink paradigm: evidence 
from human electrophysiology. Eur. J. Cog. Psychol. 18, 560-578. doi: 10.1080/09541440500423210

Kiss, M., Jolicoeur, P., Dell'Acqua, R., and Eimer, M. (2008). Attentional capture by visual singletons is mediated by top-down task set: new evidence from the N2pc component. Psychophysiology 45, 1013-1024. doi: 10.1111/j.14698986.2008.00700.x

Leblanc, E., Prime, D. J., and Jolicoeur, P. (2008). Tracking the location of visuospatial attention in a contingent capture paradigm. J. Cogn. Neurosci. 20, 657-671. doi: 10.1162/jocn.2008.20051

Lien, M. C., Ruthruff, E., Goodin, Z., and Remington, R. W. (2008). Contingent attentional capture by top-down control settings: converging evidence from event-related potentials. J. Exp. Psychol. Hum. Percept. Perform. 34, 509-530. doi: 10.1037/0096-1523.34.3.509

Martens, S., and Wyble, B. (2010). The attentional blink: past, present, and future of a blind spot in perceptual awareness. Neurosci. Biobehav. Rev. 34, 947-957. doi: 10.1016/j.neubiorev.2009.12.005

Mattler, U. (2006). On the locus of priming and inverse priming effects. Percept. Psychophys. 68, 975-991. doi: 10.3758/BF03193359

Moore, K. S., and Weissman, D. H. (2010). Involuntary transfer of a topdown attentional set into the focus of attention: evidence from a contingent attentional capture paradigm. Atten. Percept. Psychophys. 72, 1495-1509. doi: 10.3758/APP.72.6.1495

Moore, K. S., and Weissman, D. H. (2011). Set-specific capture can be reduced by preemptively occupying a limited-capacity focus of attention. Vis. Cogn. 19, 417-444. doi: 10.1080/13506285.2011.558862

Raymond, J. E., Shapiro, K. L., and Arnell, K. M. (1992). Temporary suppression of visual processing in an RSVP task: an attentional blink? J. Exp. Psychol. Hum. Percept. Perform. 18, 849-860. doi: 10.1037/0096-1523.18. 3.849

Reuss, H., Kiesel, A., Kunde, W., and Hommel, B. (2011). Unconscious activation of task sets. Conscious. Cogn. 20, 556-567. doi: 10.1016/j.concog.2011. 02.014
Serences, J. T., Shomstein, S., Leber, A. B., Golay, X., Egeth, H. E., and Yantis, S. (2005). Coordination of voluntary and stimulus-driven attentional control in human cortex. Psychol. Sci. 16, 114-122. doi: 10.1111/j.0956-7976.2005. 00791.x

Spence, C., and McDonald, J. (2004). "The cross-modal consequences of the exogenous spatial orienting of attention," in The Handbook of Multisensory Processes, eds G. A. Calvert, C. Spence and B. E. Stein (Cambridge, MA: MIT Press), 3-25.

Spence, C., McDonald, J., and Driver, J. (2004). "Exogenous spatial-cuing studies of human crossmodal attention and multisensory integration," in Crossmodal Space and Crossmodal Attention, eds C. Spence and J. Driver (New York: Oxford University Press), 277-320.

Theeuwes, J. (2010). Top-down and bottom-up control of visual selection. Acta Psychol. (Amst.) 135, 77-99. doi: 10.1016/j.actpsy.2010.02.006

Yantis, S. (2000). "Goal-directed and stimulus-driven determinants of attentional control," in Attention and Performance, eds S. Monsell and J. Driver (Cambridge, MA: MIT Press), 73-103.

Conflict of Interest Statement: The authors declare that the research was conducted in the absence of any commercial or financial relationships that could be construed as a potential conflict of interest.

Received: 25 January 2014; accepted: 13 May 2014; published online: 02 June 2014. Citation: Kamke MR and Harris J (2014) Contingent capture of involuntary visual attention interferes with detection of auditory stimuli. Front. Psychol. 5:528. doi: 10.3389/fpsyg.2014.00528

This article was submitted to Cognition, a section of the journal Frontiers in Psychology. Copyright (C) 2014 Kamke and Harris. This is an open-access article distributed under the terms of the Creative Commons Attribution License (CC BY). The use, distribution or reproduction in other forums is permitted, provided the original author(s) or licensor are credited and that the original publication in this journal is cited, in accordance with accepted academic practice. No use, distribution or reproduction is permitted which does not comply with these terms. 